\title{
Neurological function following cerebral ischemia/reperfusion is improved by the Ruyi Zhenbao pill in a rats
}

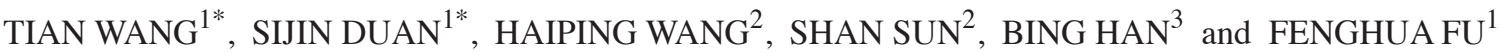 \\ ${ }^{1}$ Department of Pharmacology, School of Pharmacy, Yantai University, Yantai, Shandong 264005; \\ ${ }^{2}$ Shandong ARURA Pharmaceutical R\&D Co., Ltd., Jinan, Shandong 250101; ${ }^{3}$ Department of Food Safety, \\ School of Life Science, Yantai University, Yantai, Shandong 264005, P.R. China
}

Received April 16, 2015; Accepted December 16, 2015

DOI: $10.3892 /$ br.2016.568

\begin{abstract}
The present study aimed to investigate the effect and underlying mechanisms of the Ruyi Zhenbao pill on neurological function following cerebral ischemia/reperfusion in rats. Male Sprague-Dawley rats underwent middle cerebral artery occlusion following reperfusion. The rats received intragastrically either sodium carboxymethyl cellulose (control and model groups) or Ruyi Zhenbao pill at doses of $0.2,0.4$ or $0.8 \mathrm{~g} / \mathrm{kg}$. Neurological function was assessed by cylinder, adhesive and beam-walking tests after 14-day Ruyi Zhenbao pill treatment. Neurogenesis and angiogenesis were detected using immunofluorescence staining. The expression levels of brain-derived neurotrophic factor (BDNF), nerve growth factor (NGF) and vascular endothelial growth factor (VEGF) were determined by enzyme-linked immunosorbent assays. Treatment with 0.4 and $0.8 \mathrm{~g} / \mathrm{kg}$ Ruyi Zhenbao for 14 days significantly improved neurological function, and increased the number of von Willebrand Factor- and neuronal nuclear antigen-positive cells in the ischemic hemisphere of rats. Ruyi Zhenbao pill treatment also significantly enhanced the expression levels of BDNF, NGF and VEGF in the ischemic hemisphere. The results demonstrated that the Ruyi Zhenbao pill improved neurological function following ischemia in rats. The mechanisms of the Ruyi Zhenbao pill are associated with increasing the expression levels of BDNF, NGF and VEGF, and subsequently promoting neurogenesis and angiogenesis in the ischemic zone.
\end{abstract}

\section{Introduction}

Ischemic stroke is the second leading cause of fatality and the first leading cause of disability in adults worldwide (1). There

Correspondence to: Professor Fenghua Fu, Department of Pharmacology, School of Pharmacy, Yantai University, 32 Qingquan Road, Yantai, Shandong 264005, P.R. China

E-mail: fufenghua@sohu.com

${ }^{*}$ Contributed equally

Key words: Ruyi Zhenbao pill, neurological function, cerebral ischemia, neurogenesis, angiogenesis are currently very few effective clinical therapies for acute ischemic stroke. Administration of intravenous thrombolysis with tissue plasminogen activator (t-PA) is currently approved in numerous countries for the treatment of ischemic stroke (2). However, extremely few stroke patients benefit from this medication. Treatment with t-PA restores blood flow by recovering the oxygen and nutrient supply. However, a secondary impairment known as cerebral ischemia/reperfusion injury often occurs following t-PA treatment $(3,4)$. This ischemia/reperfusion injury induced by oxidative damage, inflammation, glutamate neurotoxicity and cerebral edema can aggravate brain damage $(5,6)$. A number of stroke patients survived with long-term neurological function impairment. Therefore, searching for effective medical treatments to improve functional recovery of post-stroke patients is of importance.

The primary neurovascular responses during stroke recovery are believed to involve angiogenesis and neurogenesis. Brain angiogenesis and neurogenesis are suggested as highly coordinated responses following ischemic brain injury (7). Following ischemic stroke, the production of neurons increased and neuroblasts migrated toward the damaged brain tissue as part of endogenous repair and brains exhibited vascular remodeling as well (8). Molecules such as vascular endothelial growth factor (VEGF), brain-derived neurotrophic factor (BDNF) and nerve growth factor (NGF) not only enhance focal angiogenesis, but also promote neurogenesis in the injured brain (9). Studies in animals also show that promoting post-ischemic angiogenesis and neurogenesis can improve the recovery of neurological function (10), which indicates it is a promising therapeutic strategy for ischemic stroke.

The Ruyi Zhenbao pill, a traditional Tibetan medicine, contains 30 types of rare medicinal herbs, including Nacre, Safflower, Musk and Cornu Bubali. The function of the Ruyi Zhenbao pill is clearing heat, opening the orifices and relaxing the sinews. The Ruyi Zhenbao pill is widely used for treating white vein, numb limbs and hemiplegia. After $24 \mathrm{~h}$ of middle cerebral artery occlusion, Ruyi Zhenbao Pian reduced the neurological deficit scores and the content of tumor necrosis factor- $\alpha$ in the ischemic hemisphere in rats (11). Clinical studies also demonstrated a beneficial effect of the Ruyi Zhenbao pill in functional recovery in stroke patients (12). Despite the evidence for the neuroprotective benefits of the Ruyi Zhenbao pill in the treatment of stroke, the effects of the Ruyi Zhenbao 
pill on angiogenesis, neurogenesis and neurological function during stroke recovery have not been investigated. In the present study, the effect of the Ruyi Zhenbao pill was investigated on the neurological function following focal cerebral ischemia/reperfusion in rats.

\section{Materials and methods}

Drug. Shandong ARURA Pharmaceutical R\&D Co., Ltd. provided the Ruyi Zhenbao pill (no. 20130225; Shandong, China).

Animal models. A total of 70 male Sprague-Dawley rats, weighing $280 \pm 20 \mathrm{~g}$, were provided by Beijing HFK Bioscience Co., Ltd. (Beijing, China), certificate number: SCXK (Beijing) 2009-0015. All the experimental procedures carried out in the study were performed in accordance with the guidelines for the Care and Use of Laboratory Animals of Yantai University (Yantai, Shandong, China). The rats had free access to food and water on a 12-h light/dark cycle.

Cerebral ischemia/reperfusion was carried out according to the method of a previous study (13). Briefly, rats were anesthetized with chloral hydrate $[300 \mathrm{mg} / \mathrm{kg}$, intraperitoneal (i.p.)]. Following the skin incision, the left common carotid artery (CCA), internal carotid artery (ICA) and external carotid artery (ECA) were dissected carefully from the surrounding nerves and fascia. CCA was ligated with a 3-0 sutures and ECA were tied loosely, but not ligated with similar sutures. A coated 6-0 filament with a rounded tip was introduced into the ICA through a small incision into the distal end of the common carotid artery, and positioned $\sim 18 \mathrm{~mm}$ from the carotid bifurcation. Subsequent to fixing the occluder in place with a suture, the ligation ECA was removed. The filament was withdrawn for reperfusion 90 min later. Parallel groups of rats received sham surgery in which an identical procedure was followed, but omitting the insertion of the filament. The rats recovered from anesthesia and were returned to their cages with free access to food and water.

Neurological assessment. A neurological examination was performed $24 \mathrm{~h}$ after cerebral ischemia/reperfusion according to Longa's methods (14) as follows: 0 , no deficit; 1 , failure to extend left forepaw fully; 2 , circling to the left; 3 , falling to the left; and 4, no spontaneous walking with a depressed level of consciousness. The rats with scores of 0 or 4 were excluded from the study.

Grouping and treatment. According to the score of neurological assessment, rats were randomly assigned to 4 groups: Model group ( $\mathrm{n}=11)$, and Ruyi Zhenbao pill at doses of $0.2,0.4$, and $0.8 \mathrm{~g} / \mathrm{kg}$ groups ( $\mathrm{n}=9)$. The rats in the Ruyi Zhenbao pill groups were orally administered with the Ruyi Zhenbao pill at doses of $0.2,0.4$, or $0.8 \mathrm{~g} / \mathrm{kg}$ once a day for 14 days, while the rats in the control $(n=9)$ and model groups were dosed with vehicle $(0.5 \%$ sodium carboxymethyl cellulose).

Behavioral testing. Animals were well handled prior to all behavioral tests to reduce stress during behavioral testing. All the equipment, as well as the testing surface, was cleaned with dilute alcohol ( $75 \%$ ethanol) and allowed to dry prior to testing each animal. The investigator conducting and scoring the behavioral tests was blinded to the experimental assignment of the animals. All the tests were performed in a quiet and dimly lit room. Each test was repeated twice and separated by $\geq 30 \mathrm{~min}$.

The cylinder test was performed as described previously (15). The rat was placed inside a glass transparent cylinder $(20 \mathrm{~cm}$ in diameter and $30 \mathrm{~cm}$ in height). A mirror was placed behind the cylinder with an angle to enable the investigator to record forelimb movements when the rat was turned away. Subsequent to placing the rat in the cylinder, the forelimb use of the first contact against the wall following rearing and during lateral exploration was recorded by the following criteria: i) The first forelimb to contact the wall during a full rear was recorded as an independent wall placement for that limb. ii) Simultaneous use of both the left and right forelimb by contacting the wall of the cylinder during a full rear and for lateral movements along the wall was recorded as 'both' movement. iii) After the first forelimb (for example right forelimb) contacted the wall and subsequently the other forelimb was placed on the wall, but the right forelimb was not removed from the wall, a 'right forelimb independent' movement and a 'both' movement were recorded. However, if the other (left forelimb) made several contacting movements on the wall, a 'right forelimb independent' movement and only one 'both' movement was recorded. iv) When the rat explored the wall laterally, alternating both forelimbs, it was recorded as a 'both' movement. A total of 20 movements were recorded during the test (16). The asymmetry score was calculated using the formula: $100 \mathrm{x}$ (ipsilateral forelimb use $+1 / 2$ both)/total forelimb use observations (17).

An adhesive test was performed to assess the sensorimotor deficit, as previously described (18). Adhesive tape $(6 \mathrm{~mm}$ in diameter) was placed on the palmar surface of the paw with equal pressure and the rat was observed in a cage. The time first contact of adhesive tape was recorded for each forelimb. The rats were provided a maximum of $2 \mathrm{~min}$ to perform this task.

A beam-walking test was applied to assess the coordination and integration of motor movements (19). Rats were trained twice daily for 2 days before cerebral ischemia/reperfusion. The apparatus consisted of a wooden beam $(2.5 \times 120 \mathrm{~cm}$, positioned at a height of $50 \mathrm{~cm}$ by 2 supporting legs). A wooden box $(30 \times 30 \times 30 \mathrm{~cm})$ was set at another side motivating the animal to cross the beam. During the training session, a protocol was followed to facilitate the training of beam-walking performance: During the first trial, the animal was placed at the middle point of the testing distance; the second trial began at the starting point of the testing distance (20). The apparatus was placed above a cushion, which protected the animals against a fall injury. The time recording began when the rat extended a forepaw from the platform onto the beam to start the traverse and stopped when a forepaw was crossing the beam forward into the black box (19). The time taken to traverse the beam was recorded by a stopwatch. The cut-off time was taken as $60 \mathrm{sec}(21)$.

Histological and immunohistochemical assessment. Following the last behavioral test, 3 rats in each group were anesthetized with chloral hydrate $(300 \mathrm{mg} / \mathrm{kg}$, i.p.), and the brains were perfusion-fixed with $4 \%$ paraformaldehyde following a heparinized saline flush. The brains were subsequently dissected and post-fixed in paraformaldehyde overnight at $4^{\circ} \mathrm{C}$, after which 
A

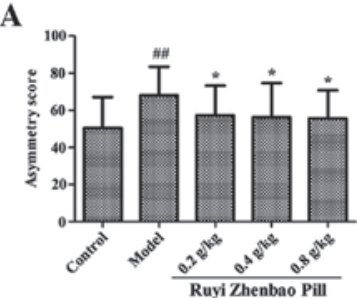

B

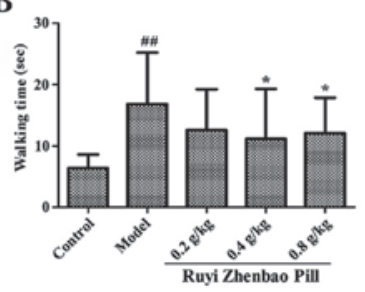

C

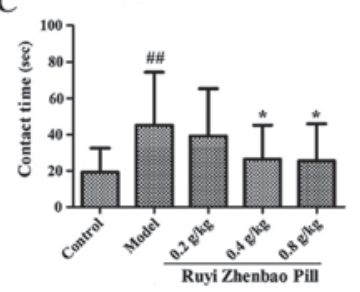

Figure 1. Effect of the Ruyi Zhenbao pill on neurological function following cerebral ischemia/reperfusion in rats. (A) Cylinder test; (B) adhesive test; and (C) beam-walking test. ${ }^{\# \#} \mathrm{P}<0.01$ vs. control group. ${ }^{*} \mathrm{P}<0.05$ vs. model group.

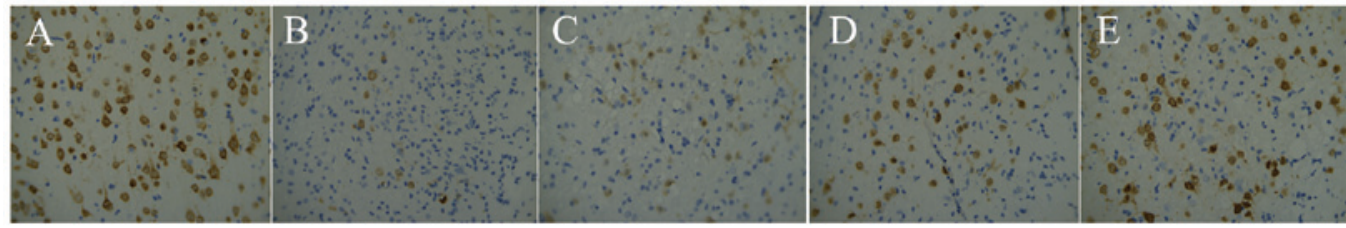

Figure 2. Neuronal nuclear antigen-immunoreactive positive neurons stained using immunocytochemistry staining in the ischemic hemisphere. (A) Control; (B) model; and (C) Ruyi Zhenbao pill at 0.2; (D) 0.4 ; and (E) $0.8 \mathrm{~g} / \mathrm{kg}$ (magnification, $\mathrm{x} 400$ ).

they were transferred into $20 \%$ sucrose in $0.1 \mathrm{M}$ phosphate buffer at $4^{\circ} \mathrm{C}$ for $24 \mathrm{~h}$. Paraffin-embedded specimens were cut at $5-\mu \mathrm{m}$ sections using a vibratome and subjected to immunohistochemistry. The brain sections were rinsed in phosphate-buffered saline with Triton X-100, quenched in 3\% hydrogen peroxide and incubated in blocking solution. After incubation with the anti-neuronal nuclear antigen (NeuN) (1:200; cat. no. MAB377; Millipore, Billerica, MA, USA) and anti-von Willebrand Factor (vWF) (1:200; cat. no. A0082; Dako, Carpinteria, CA, USA) antibody at $4^{\circ} \mathrm{C}$ for $24 \mathrm{~h}$, the sections were treated with biotinylated secondary antibody for $1 \mathrm{~h}$ at $37^{\circ} \mathrm{C}$, followed by incubation with streptavidin peroxidase for $1 \mathrm{~h}$ at $37^{\circ} \mathrm{C}$. The treated sections were incubated for $5 \mathrm{~min}$ with 3,3'-diaminobenzidine. The results were analyzed by counting the number of positive cells on an Olympus microscope (magnification, $\mathrm{x} 400$ ) in the ischemic hemisphere of rats (IX-70; Olympus Corp., Tokyo, Japan).

Enzyme-linked immunosorbent assay. The left hemisphere of the brain tissues of rats was obtained following the last behavioral test. Samples were homogenized in 4 volumes of normal saline and were centrifuged at $4,500 \mathrm{x}$ g for $15 \mathrm{~min}$ at $4^{\circ} \mathrm{C}$. The protein concentration in the supernatants was determined by the bicinchoninic acid protein assay (Beyotime Institute of Biotechnology, Hangzhou, China). The levels of VEGF, BDNF and NGF in the supernatants were assayed using commercially available kits (Shanghai Westang Bio-Tech Co., Ltd., Shanghai, China) according to the manufacturer's protocol. Duplicate samples were analyzed for each data point.

Statistical analysis. Data are presented as mean \pm standard deviation. The data were determined using one-way analysis of variance followed by least significant difference testing. $\mathrm{P}<0.05$ was considered to indicate a statistically significant difference.

\section{Results}

Effect of the Ruyi Zhenbao pill on neurological function following cerebral ischemia/reperfusion in rats. Animals in

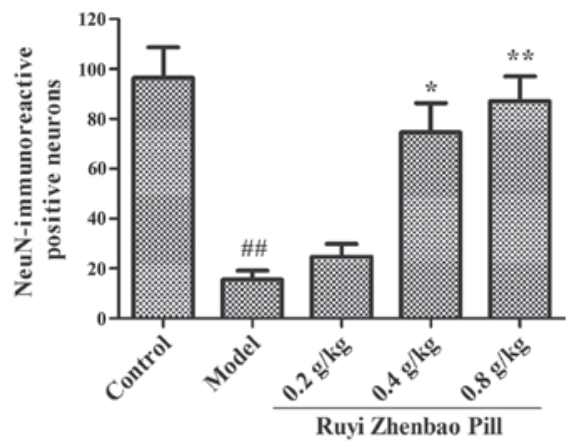

Figure 3.Effect of the RuyiZhenbao pill on the number of NeuN-immunoreactive positive neurons in the ischemic hemisphere. ${ }^{\# \#} \mathrm{P}<0.01$ vs. control group. ${ }^{*} \mathrm{P}<0.05,{ }^{* *} \mathrm{P}<0.01$ vs. model group. NeuN, neuronal nuclear antigen.

the control group did not show any paw preference during rearing in the cylinder test. The cerebral ischemia/reperfusion injury led to a motor deficit in the impaired (right) forepaw, which was indicated by a significant preference in use of the unimpaired (left) forepaw compared with the control group $(\mathrm{P}<0.01)$. Compared with the model group, treatment with $0.2,0.4$, or $0.8 \mathrm{~g} / \mathrm{kg}$ Ruyi Zhenbao improved the performance of the rats subjected to cerebral ischemia/reperfusion in the cylinder test $(\mathrm{P}<0.05)$ (Fig. 1A).

Compared with the control group, the animals in the model group spent a longer time crossing the beam $(\mathrm{P}<0.01)$. The time crossing the beam of the rats in the Ruyi Zhenbao pill $(0.4$ or $0.8 \mathrm{~g} / \mathrm{kg}$ ) groups were significantly shorter than that of the animals in the model group $(\mathrm{P}<0.05)$ (Fig. 1B).

The functional recovery of sensorimotor impairments was assessed by the time required to contact the adhesive tape from the impaired forepaw. Compared with the control group, rats in the model group had a prolonged contact time $(\mathrm{P}<0.01)$. However, significant improvements on the adhesive test were detected in rats treated with 0.4 or $0.8 \mathrm{~g} / \mathrm{kg}$ Ruyi Zhenbao compared with the rats of the model group $(\mathrm{P}<0.05)$ (Fig. 1C). 

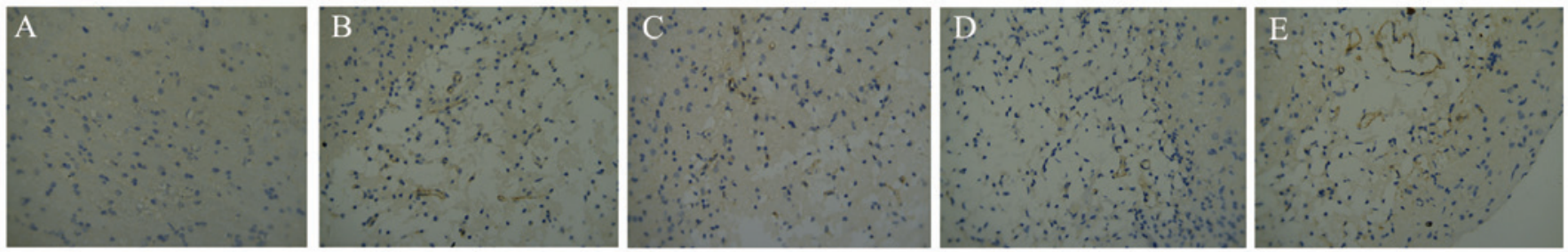

Figure 4. von Willebrand Factor-immunoreactive positive vessels stained using immunocytochemistry staining in the ischemic hemisphere. (A) Control; (B) model; and (C) Ruyi Zhenbao pill at 0.2; (D) 0.4; and (E) $0.8 \mathrm{~g} / \mathrm{kg}$ (magnification, $\mathrm{x} 400$ ).

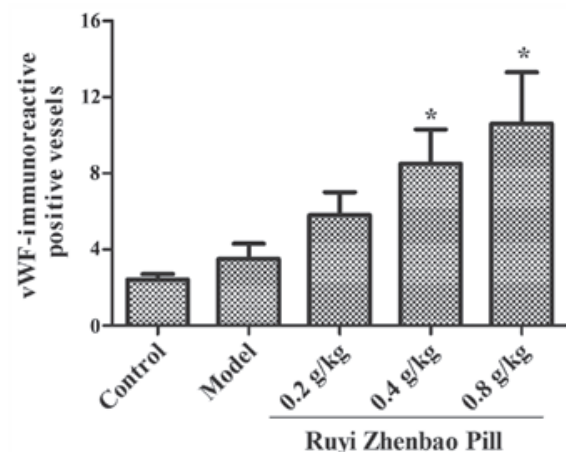

Figure 5. Effect of the Ruyi Zhenbao pill on the number of vWF-immunoreactive positive vessels in the ischemic hemisphere. ${ }^{\# \#} \mathrm{P}<0.01$ vs. control group. ${ }^{*} \mathrm{P}<0.05,{ }^{* *} \mathrm{P}<0.01$ vs. model group. vWF, von Willebrand Factor.

Effect of the Ruyi Zhenbao pill on NeuN expression. In order to elucidate the mechanism of the Ruyi Zhenbao pill improving neurological function following cerebral ischemia/reperfusion in rats, immunohistochemistry was further performed with NeuN. The quantity of NeuN-immunoreactive-positive neurons in the model decreased significantly compared with the control group; however, the quantity in the ischemic hemisphere significantly increased following treatment with the Ruyi Zhenbao pill $(\mathrm{P}<0.05)$ (Figs. 2 and 3).

Effect of the Ruyi Zhenbao pill on the expression of $v W F$. $\mathrm{vWF}$ is a specific marker for endothelial cells. In the present study, vWF-immunoreactive cells on the ischemic hemisphere were analyzed 14 days after cerebral ischemia/reperfusion. In the rats of the model group, an increase in the vWF-positive vessels was observed in the ischemic hemisphere compared with the control group. The number of vWF-positive vessels in the Ruyi Zhenbao pill ( 0.4 or $0.8 \mathrm{~g} / \mathrm{kg}$ ) groups was more than that in model group $(\mathrm{P}<0.05)$ (Figs. 4 and 5).

Effect of the Ruyi Zhenbao pill on the levels of VEGF, BDNF and NGF in the left hemisphere of the brain. The levels of VEGF, BDNF and NGF did not show significant differences in the left hemisphere of the brain of model mice compared to those in the control group. Compared with the model group, the VEGF, BDNF and NGF levels in the Ruyi Zhenbao pill groups were increased $(\mathrm{P}<0.05)$ (Fig. 6).

\section{Discussion}

The present study showed the beneficial effect of Ruyi Zhenbao pill therapy on the neurological function following cerebral
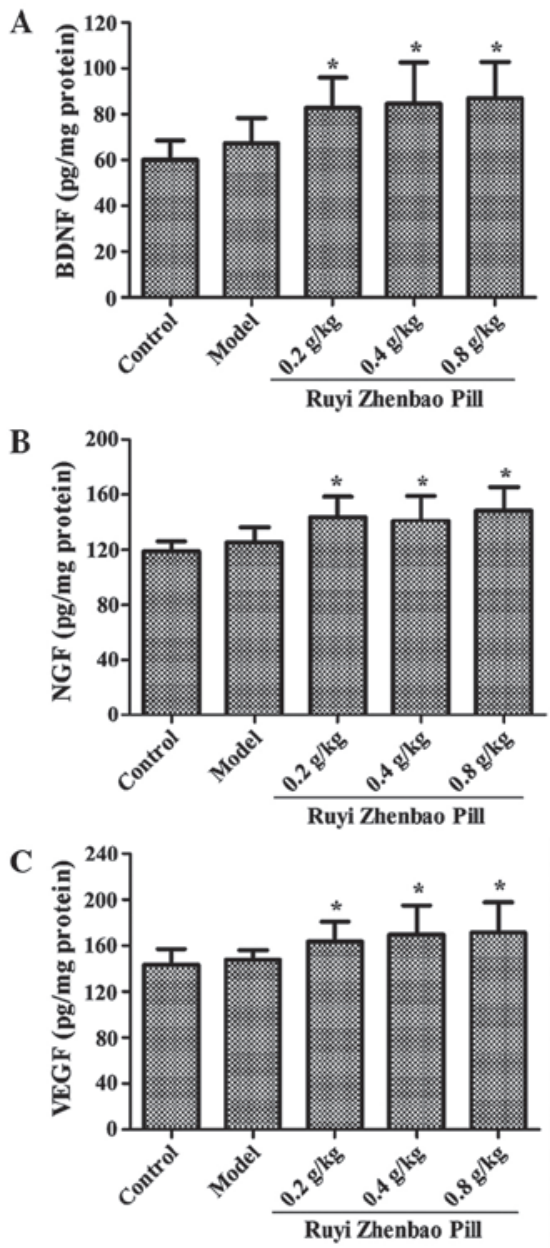

Figure 6. Effect of the Ruyi Zhenbao pill on the levels of (A) BDNF, (B) NGF and $(\mathrm{C})$ VEGF in the left hemisphere of brain. ${ }^{*} \mathrm{P}<0.05$ vs. model group. BDNF, brain-derived neurotrophic factor; NGF, nerve growth factor; VEGF, vascular endothelial growth factor.

ischemia/reperfusion in rats. The Ruyi Zhenbao pill improved the neurological function after cerebral ischemia/reperfusion at 14 days following Ruyi Zhenbao pill treatment and promoted angiogenesis and neurogenesis in the ischemic hemisphere of brain at the same time. Increases of the VEGF, BDNF and NGF levels were also identified in the ischemic hemisphere of brain, which suggested that the effect of angiogenesis and neurogenesis by the Ruyi Zhenbao pill was via regulation of VEGF, BDNF and NGF levels.

The Ruyi Zhenbao pill is a traditional Tibetan medicine. Clinical studies have demonstrated a beneficial effect of the Ruyi Zhenbao pill in the functional recovery in stroke patients. 
In order to evaluate the effect of the Ruyi Zhenbao pill on the neurological function following stroke, cerebral ischemia/reperfusion animals were used as a stroke model. Notably, this model is considered to mimic focal ischemia-induced stroke in human (22). In the present study, the Ruyi Zhenbao pill improved the neurological function and promoted focal angiogenesis and neurogenesis during focal cerebral ischemia. In addition, the Ruyi Zhenbao pill increased the VEGF, BDNF, and NGF levels in the ischemic hemisphere during ischemia. To the best of our knowledge, this is the first study to demonstrate that treatment with the Ruyi Zhenbao pill following ischemia could promote angiogenesis and neurogenesis, which identified a potential candidate for future stroke therapy.

Functional disabilities following ischemic stroke are often prominent and therefore it is of fundamental importance to investigate changes in animal behavior following cerebral ischemia (23). In the present study, sensorimotor functions were measured by the cylinder test, beam-walking test and adhesive test. The cylinder test is a standard test to investigate motor impairment following ischemic brain injury (24). It has been applied widely to evaluate possible motor impairments caused by stroke due to its simplicity, reliability and reproducibility (25). The other behavioral recovery was assessed with the beam-walking test to detect motor coordination recovery following stroke. The beam-walking test assesses motor ability that requires muscle strength, coordination of flexor and extensor muscles, and integration of sensory information (26). Particular interest was given to the adhesive tests, which proved to be useful to detect long-term deficits following stroke in rats (27-29). In the present study, the adhesive test was used to evaluate the recovery in sensory function. The assessment of animal behavior showed that cerebral ischemia/reperfusion caused the impairments of motor function, motor coordination, and sensory function in rats. However, the Ruyi Zhenbao pill treatment increased the usage of contralateral forelimb and reduced the time of crossing the beam, and the time required to contact the adhesive tape.

In the normal brain, the occurrence of neurogenesis and angiogenesis usually accompany each other, which is important for the formation of neurovascular unit. Following ischemic brain injury, this correlation still exist (8). The conditions that enhance focal angiogenesis and neurogenesis exerted valuable function via promoting functional recovery (30). The present study detected an increase in the number of NeuN-positive cells and vWF-positive vessels, suggesting that the Ruyi Zhenbao pill could promote angiogenesis and neurogenesis during the reperfusion period.

BDNF and NGF are members of the neurotrophin family protein that exert strong survival and differentiation functions during the development of the nervous system (31). BDNF and NGF also represent a crucial signaling molecule in adaptive brain plasticity following stroke (32). Interventions that improve recovery of function are most often associated with increased BDNF and NGF levels in perilesional areas (33). Conversely, attenuating BDNF and NGF levels or its effects following cerebral ischemia reduces neuroplastic changes or recovery of function either spontaneously or induced by rehabilitation (34). From these data, pharmacological strategies aimed at increasing post-ischemic cerebral BDNF and NGF production appear to be a promising option in the treatment of stroke.
VEGF is an angiogenic and vascular permeability factor, which also has neurotrophic, neuroprotective and neuroproliferative effects (35). The ability of VEGF to elicit angiogenesis and neuroprotection has led to interest in its possible therapeutic application in stroke. The present study demonstrated that the recovery of neurological function in the stroke rats induced by Ruyi Zhenbao pill treatment is mediated by the increase of BDNF, VEGF and NGF. The present study demonstrates for the first time that the Ruyi Zhenbao pill increase BDNF, VEGF and NGF expression, and improves neurological function in a stroke model. This finding has an important role in understanding the mechanism of action of the Ruyi Zhenbao pill in the neurological functional recovery following stroke.

In conclusion, the results demonstrated that the Ruyi Zhenbao pill improved neurological function following ischemia in rats. The mechanism of the Ruyi Zhenbao pill is associated with increasing the expression levels of BDNF, NGF and VEGF, and subsequently promoting neurogenesis and angiogenesis in the ischemic zone.

\section{Acknowledgements}

The study was supported by the service platform of the Innovation of Industrial Technology in Tibetan Medicine, the National Natural Science Foundation of China (grant no. 81274125), the Taishan Scholar Project (Professor Fenghua $\mathrm{Fu}$ ) and the project of star of Youth in Science and Technology (grant no. 2013019).

\section{References}

1. Johnston SC, Mendis S and Mathers CD: Global variation in stroke burden and mortality: Estimates from monitoring, surveillance and modelling. Lancet Neurol 8: 345-354, 2009.

2. Young AR, Ali C, Duretête A and Vivien D: Neuroprotection and stroke: Time for a compromise. J Neurochem 103: 1302-1309, 2007.

3. Wong $\mathrm{CH}$ and Crack PJ: Modulation of neuro-inflammation and vascular response by oxidative stress following cerebral ischemia-reperfusion injury. Curr Med Chem 15: 1-14, 2008.

4. Eltzschig HK and Eckle T: Ischemia and reperfusion-from mechanism to translation. Nat Med 17: 1391-1401, 2011.

5. Huang J, Upadhyay UM and Tamargo RJ: Inflammation in stroke and focal cerebral ischemia. Surg Neurol 66: 232-245, 2006.

6. Gürsoy-Ozdemir Y, Can A and Dalkara T: Reperfusion-induced oxidative/nitrative injury to neurovascular unit after focal cerebral ischemia. Stroke 35: 1449-1453, 2004.

7. Arai K, Jin G, Navaratna D and Lo EH: Brain angiogenesis in developmental and pathological processes: Neurovascular injury and angiogenic recovery after stroke. FEBS J 276: 4644-4652, 2009.

8. Moskowitz MA, Lo EH and Iadecola C: The science of stroke: Mechanisms in search of treatments. Neuron 67: 181-198, 2010.

9. Raab S and Plate KH: Different networks, common growth factors: Shared growth factors and receptors of the vascular and the nervous system. Acta Neuropathol 113: 607-626, 2007.

10. Yao RQ, Zhang L, Wang W and Li L: Cornel iridoid glycoside promotes neurogenesis and angiogenesis and improves neurological function after focal cerebral ischemia in rats. Brain Res Bull 79: 69-76, 2009.

11. Gu LJ and Zhang J: Protective effect of Ruyi Zhenbao pian on experimental cerebral ischemia in rats. Chin Arch Tradi Chin Med 31: 1288-1290, 2013 (In Chinese).

12. Liu X, Wang L and Fu J: To study the effect of Ruyi Zhenbao pill on functional recovery of post-stroke patients. Shandong Medi 50: 88, 2010 (In Chinese).

13. Pereira BM and Weinstein PR, Zea-Longa E and el-Fiki M: Effect of blood flow rate and donor vessel diameter on the patency of carotid venous bypass grafts in dogs. Surg Neurol 31: 195-199, 1989. 
14. Longa EZ, Weinstein PR, Carlson S and Cummins R: Reversible middle cerebral artery occlusion without craniectomy in rats Stroke 20: 84-91, 1989.

15. Shi GX, Yang CY, Wu MM, Guan LP, Wang LP and Liu CZ: Muscle hypertonia after permanent focal cerebral ischemia in rats: A qualitative and quantitative behavioral and electrophysiological study. Int J Neurosci 123: 575-581, 2013.

16. Soleman S, Yip P, Leasure JL and Moon L: Sustained sensorimotor impairments after endothelin-1 induced focal cerebral ischemia (stroke) in aged rats. Exp Neurol 222: 13-24, 2010.

17. Allred RP, Maldonado MA, Hsu Je and Jones TA: Training the 'less-affected' forelimb after unilateral cortical infarcts interferes with functional recovery of the impaired forelimb in rats. Restor Neurol Neurosci 23: 297-302, 2005.

18. Schallert T, Upchurch M, Wilcox RE and Vaughn DM Posture-independent sensorimotor analysis of inter-hemispheric receptor asymmetries in neostriatum. Pharmacol Biochem Behav 18: 753-759, 1983.

19. Lin B, Levy S, Raval AP, Perez-Pinzon MA and Defazio RA Forebrain ischemia triggers GABAergic system degeneration in substantia nigra at chronic stages in rats. Cardiovasc Psychiatry Neurol 2010: 506952, 2010.

20. Holmberg P, Liljequist S and Wägner A: Secondary brain injuries in thalamus and hippocampus after focal ischemia caused by mild, transient extradural compression of the somatosensor cortex in the rat. Curr Neurovasc Res 6: 1-11, 2009.

21. Moro N and Sutton RL: Beneficial effects of sodium or ethyl pyruvate after traumatic brain injury in the rat. Exp Neurol 225 : 391-401, 2010

22. Lyden PD, Zivin JA, Chabolla DR, Jacobs MA and Gage FH: Quantitative effects of cerebral infarction on spatial learning in rats. Exp Neurol 116: 122-132, 1992

23. Porritt MJ, Andersson HC, Hou L, Nilsson A, Pekna M, Pekny M and Nilsson M: Photothrombosis-induced infarction of the mouse cerebral cortex is not affected by the Nrf2-activator sulforaphane. PLoS One 7: e41090, 2012.

24. Schallert T, Fleming SM, Leasure JL, Tillerson JL and Bland ST: CNS plasticity and assessment of forelimb sensorimotor outcome in unilateral rat models of stroke, cortical ablation, parkinsonism and spinal cord injury. Neuropharmacology 39: 777-787, 2000.

25. Chang YS, Mu D, Wendland M, Sheldon RA, Vexler ZS, McQuillen PS and Ferriero DM: Erythropoietin improves functional and histological outcome in neonatal stroke. Pediat Res 58: 106-111, 2005.
26. Nomura S, Kagawa Y, Kida H, Maruta Y, Imoto H, Fujii M and Suzuki M: Effects of intrathecal baclofen therapy on motor and cognitive functions in a rat model of cerebral palsy. J Neurosurg Pediatr 9: 209-215, 2012.

27. Park SI, Jang DK, Han YM, Sunwoo YY, Park MS, Chung YA, Maeng LS, Im R, Kim MW, Jeun SS and Jang KS: Effect of combination therapy with sodium ozagrel and panax ginseng on transient cerebral ischemia model in rats. J Biomed Biotechnol 2010: 893401, 2010

28. Gow WR, Campbell K, Meade AJ, Watt PM, Milech N, Knuckey NW and Meloni BP: Lack of neuroprotection of inhibitory peptides targeting Jun/JNK after transient focal cerebral ischemia in spontaneously hypertensive rats. J Cereb Blood Flow Metab 31: e1-e8, 2011.

29. Liu YP, Seçkin H, Izci Y, Du ZW, Yan YP and Başkaya MK: Neuroprotective effects of mesenchymal stem cells derived from human embryonic stem cells in transient focal cerebral ischemia in rats. J Cereb Blood Flow Metab 29: 780-791, 2009.

30. Wang L, Zhang Z, Wang Y, Zhang R and Chopp M: Treatment of stroke with erythropoietin enhances neurogenesis and angiogenesis and improves neurological function in rats. Stroke 35: 1732-1737, 2004.

31. Huang EJ and Reichardt LF: Neurotrophins: Roles in neuronal development and function. Annu Rev Neurosci 24: 677-736, 2001.

32. Cowansage KK, LeDoux JE and Monfils MH: Brain-derived neurotrophic factor: A dynamic gatekeeper of neural plasticity. Curr Mol Pharmacol 3: 12-29, 2010.

33. Kim MW, Bang MS, Han TR, Ko YJ, Yoon BW, Kim JH, Kang LM, Lee KM and Kim MH: Exercise increased BDNF and trkB in the contralateral hemisphere of the ischemic rat brain. Brain Res 1052: 16-21, 2005

34. Madinier A, Bertrand N, Mossiat C, Prigent-Tessier A, Beley A, Marie C and Garnier P: Microglial involvement in neuroplastic changes following focal brain ischemia in rats. PLoS One 4: e8101, 2009.

35. Greenberg DA and Jin K: From angiogenesis to neuropathology. Nature 438: 954-959, 2005. 